\title{
Role of multi-detector computed tomography in congenital heart diseases
}

\author{
Duong Phi Son ${ }^{1 *}$, Nguyen Tuan Vu ${ }^{2}$ and Phan Thanh Hai ${ }^{1}$ \\ ${ }^{1}$ Medic Medical Center, Ho Chi Minh City, Vietnam \\ ${ }^{2}$ Pham Ngoc Thach Medical University, Ho Chi Minh City, Vietnam
}

\begin{abstract}
Background: Congenital heart diseases associated with more malformations, complex aortopulmonary collaterals and anomalous coronary artery.

Echocardiography is the initial diagnostic method but this method can be limited in complex congenital heart diseases.

Purpose: To assess the role of MDCT in congenital heart diseases (CHD) diagnosis compare with operative result and interventional angiography.

Methods: 910 patients with congenital heart diseases of 31.000 patients underwent cardiac angiography with 64 and 320 section CT at Medic Medical Center since 09/09/2006 to $30 / 12 / 2015$.

Results: There are 658 operated cases, most of operated cases demonstrated the exact diagnosis of MDCT in congenital heart diseases.

Conclusions: MDCT is the fast and non-invasive diagnostic method with the high accuracy, overcomes the limit of echocardiography in complex congenital heart diseases diagnosis and provides the panorama and useful informations prior to the operation.
\end{abstract}

\section{Introduction}

Congenital heart diseases effect $\sim 1 \%$ of all live births in the general population. Complex congenital heart diseases associated with more malformations, complex aortopulmonary collaterals and anomalous coronary artery.

Over the past few decades, the diagnosis and treatment of congenital heart diseases have greatly improved [1-4]. Diagnostic tools: X-ray, ECG, echocardiography, MRI and MDCT. ECG and X-Ray suggest the diagnosis but are not specific. Echocardiography is the initial diagnostic method for patients with suspected CHD but this method can be limited in complex CHD.

The great capabilities of MRI for anatomic and functional assessment of the heart but MRI is time-consuming and may require patient sedation.

Now enable CT to be used as an accurate non-invasive clinical instrument that is fast replacing invasive cine-angiography in the evaluation of CHD $[1,5,6]$ :

- Improves both spatial and temporal resolution.

- Increases scanning speed.

- Improves diagnostic image quality by reducing respiratory artifacts

\section{Purpose}

To assess the role of MDCT in congenital heart diseases (CHD) diagnosis compare with operative result and interventional angiography

\section{Material and methods}

\section{Subject}

910 patients with congenital heart diseases of 31.000 patients underwent cardiac angiography with 64 and 640 section CT at Medic Medical Center since 09/09/2006 to 30/12/2015.

\section{Means and scanning techniques}

Medic Medical Center scanned cardiac CT by 64 MDCT Toshiba Aquilion machine and Toshiba Aquilion One (320 MDCT), $0.5 \mathrm{~mm}$ slice thickness, $0.5 \mathrm{~mm}$ imaging reconstruction.

- Two phases scanning (Figure 1): Don't inject phase and contrast media injection phase:

$>\quad$ Phase doesn't inject contrast which help locate and assess coronary artery calcification.

$>\quad$ Phase inject contrast media: Medicine chasing phase and water chasing phase.

- Contrast pumping machine is double-barreled Stellant (Medrad).

${ }^{\star}$ Correspondence to: Son DP, Medic Medical Center, Ho Chi Minh City, Vietnam, E-mail: drphison0912@gmail.com

Key words: congenital heart diseases, cardiac multi-detector computed tomography, multi-detector computed tomography in congenital heart diseases, congenital heart diseases computed tomography

Received: July 01, 2019; Accepted: July 08, 2019; Published: July 11, 2019 


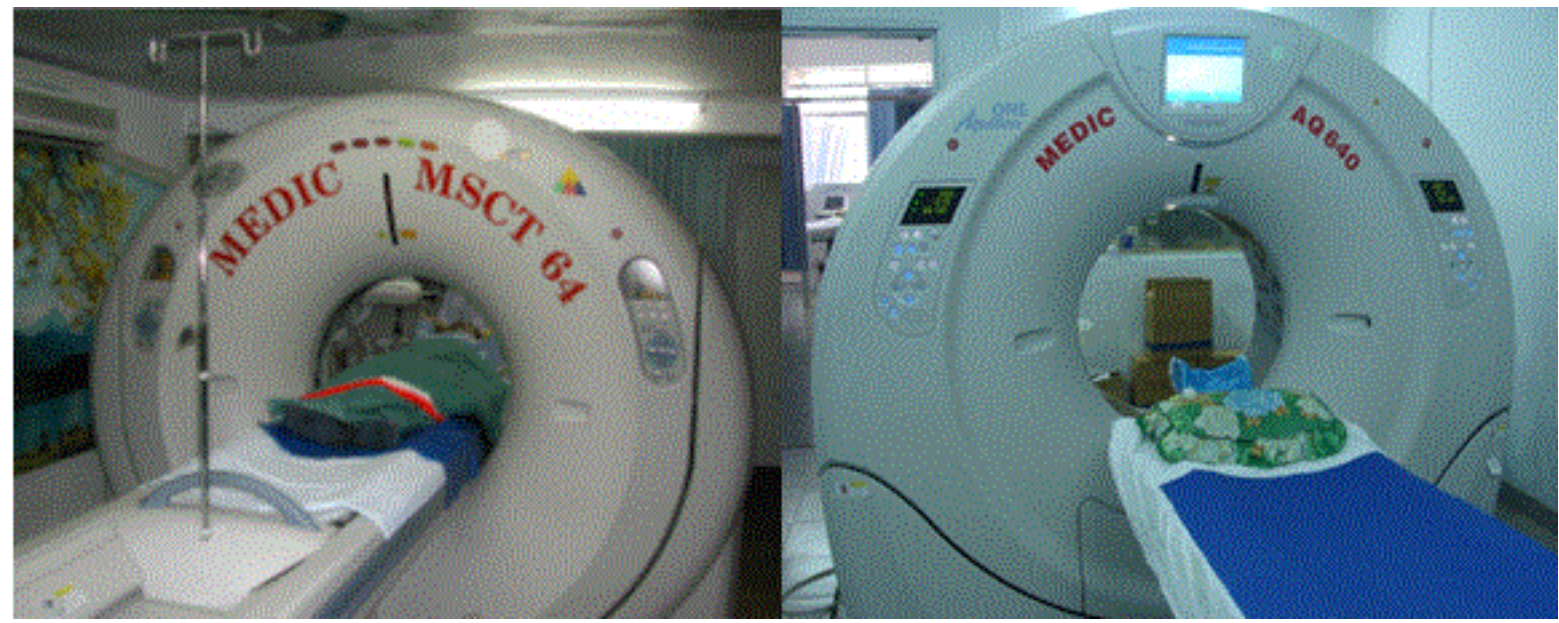

Figure 1. Two CT scanner

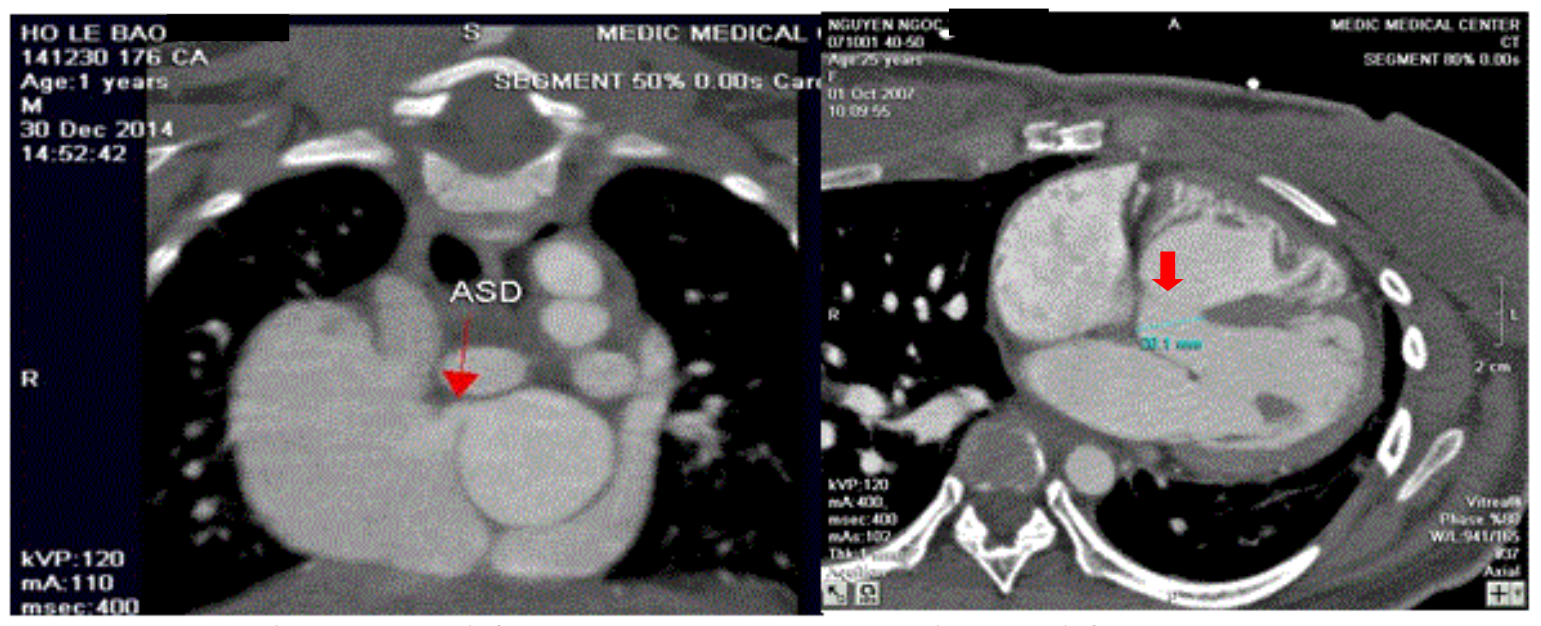

Sinus venosus defect

Membranous defect

Figure 2. Atrial septal defects and Ventricular septal defects.

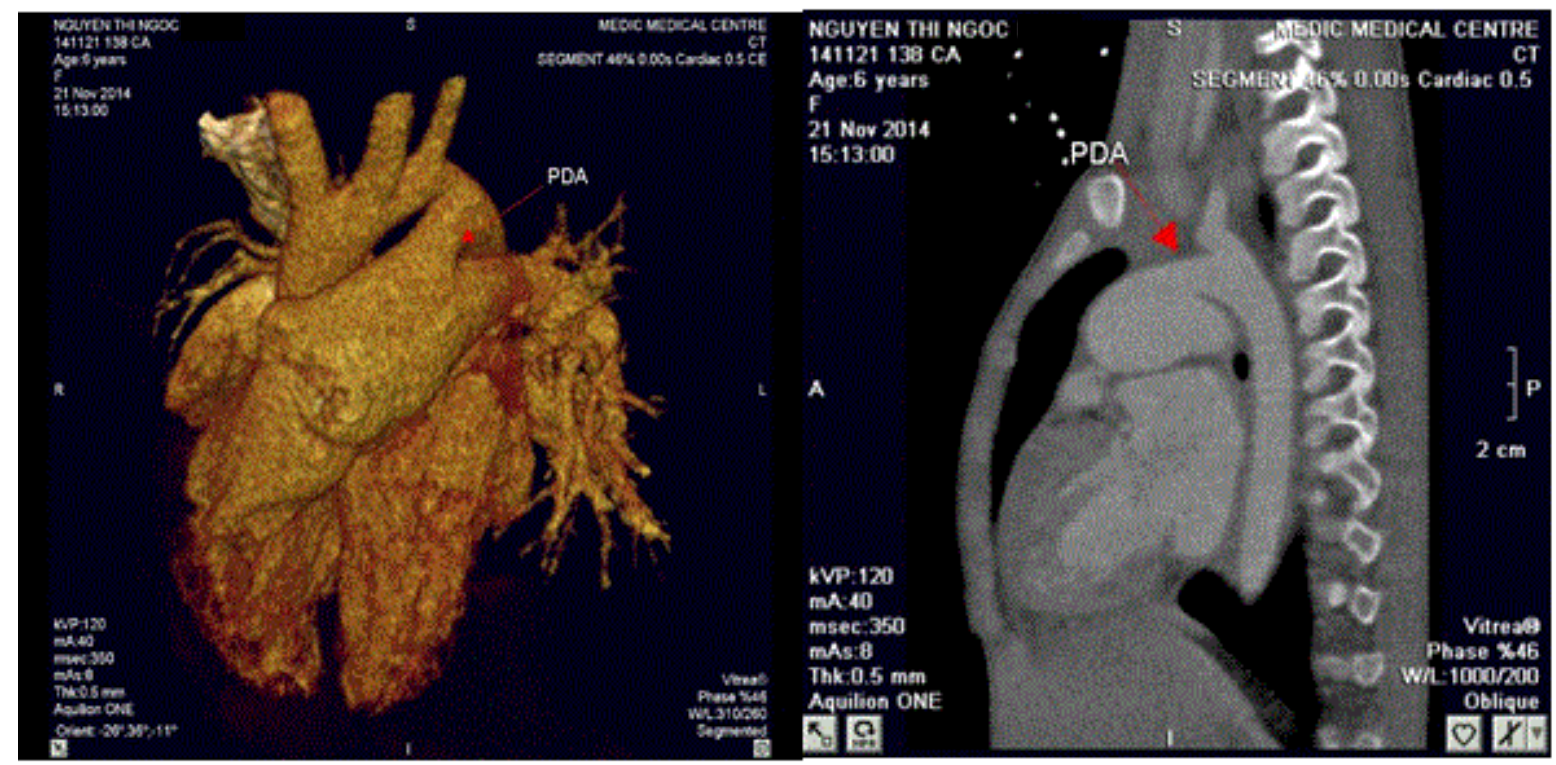

Figure 3. Patent ductus arteriosus; 3D images: Patent ductus arteriosus 


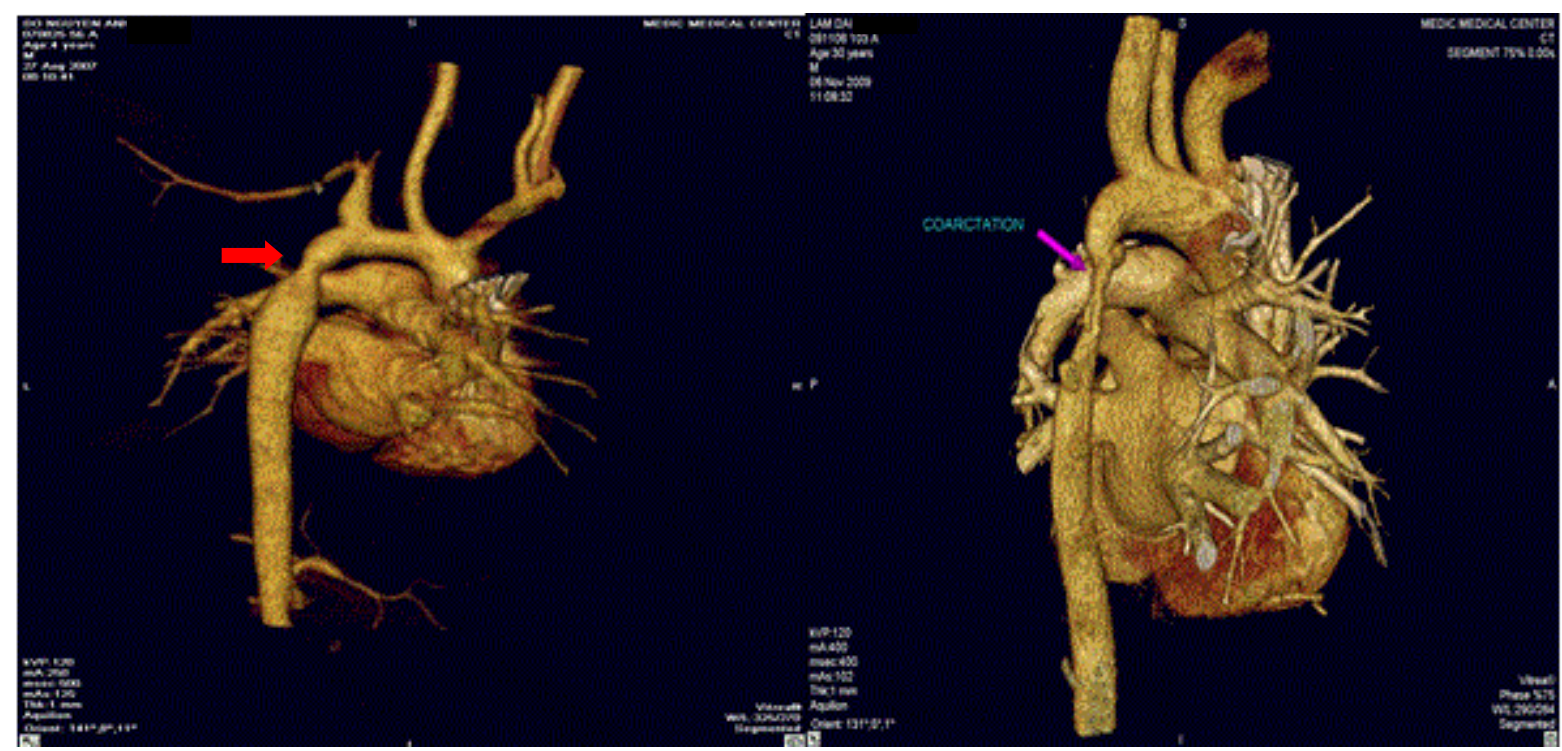

Figure 4. Coarctation of aorta

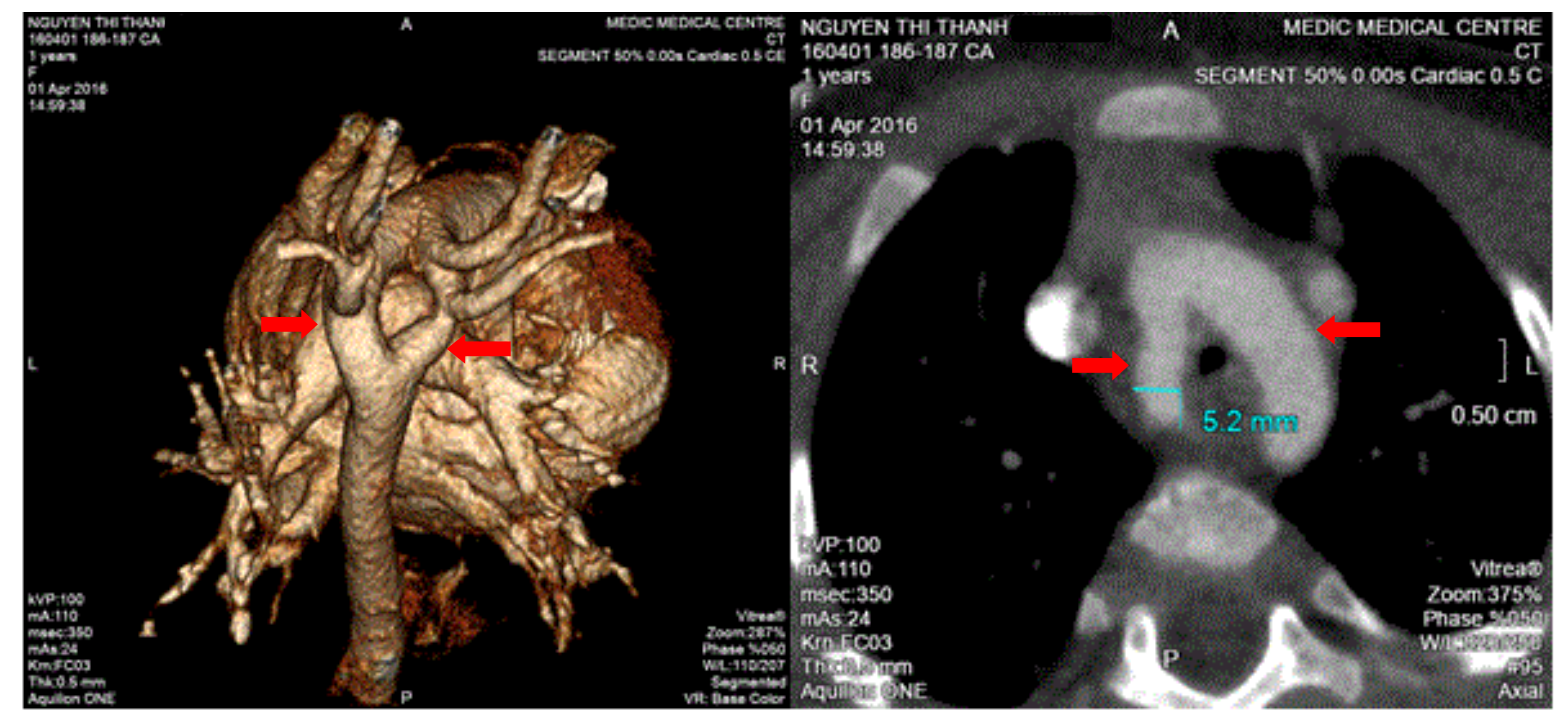

Figure 5. Double aortic arch; 3D images and Axial: Contrast-enhanced CT double aortic arch

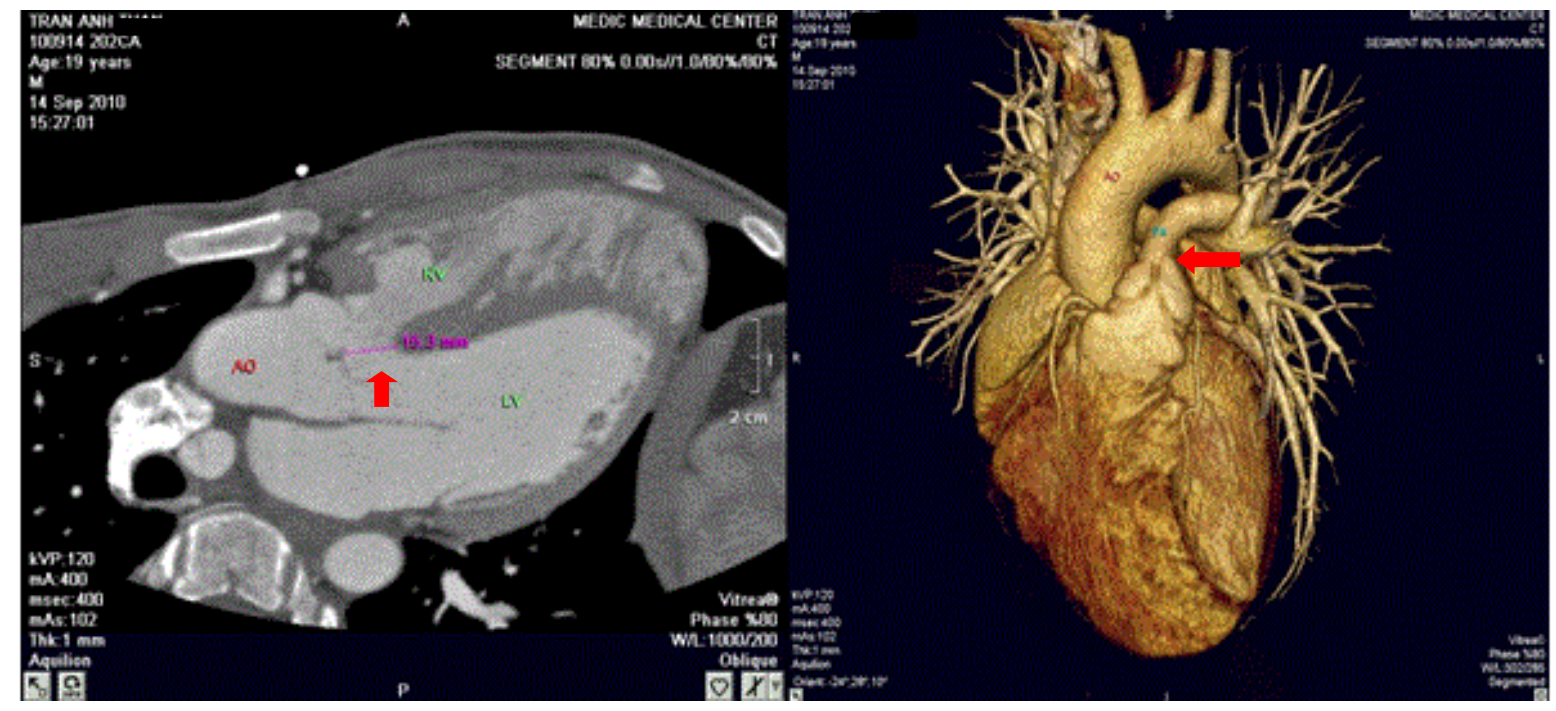

Figure 6. Tetralogy of Fallot; Subaortic ventricular septal defect, over riding aorta and pulmonary artery stenosis 


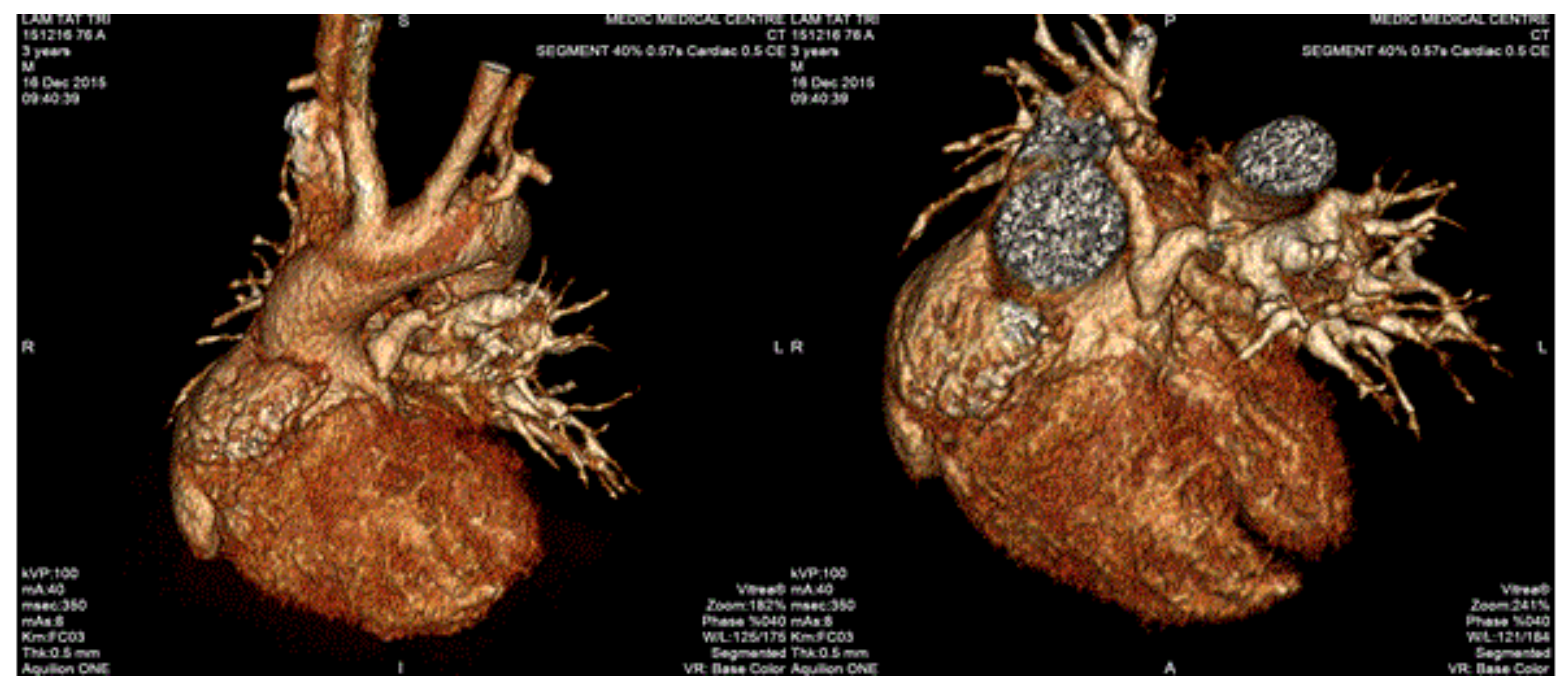

Figure 7. Pulmonary atresia with ventricular septal defect; Pulmonary atresia with ventricular septal defect and confluence of pulmonary artery

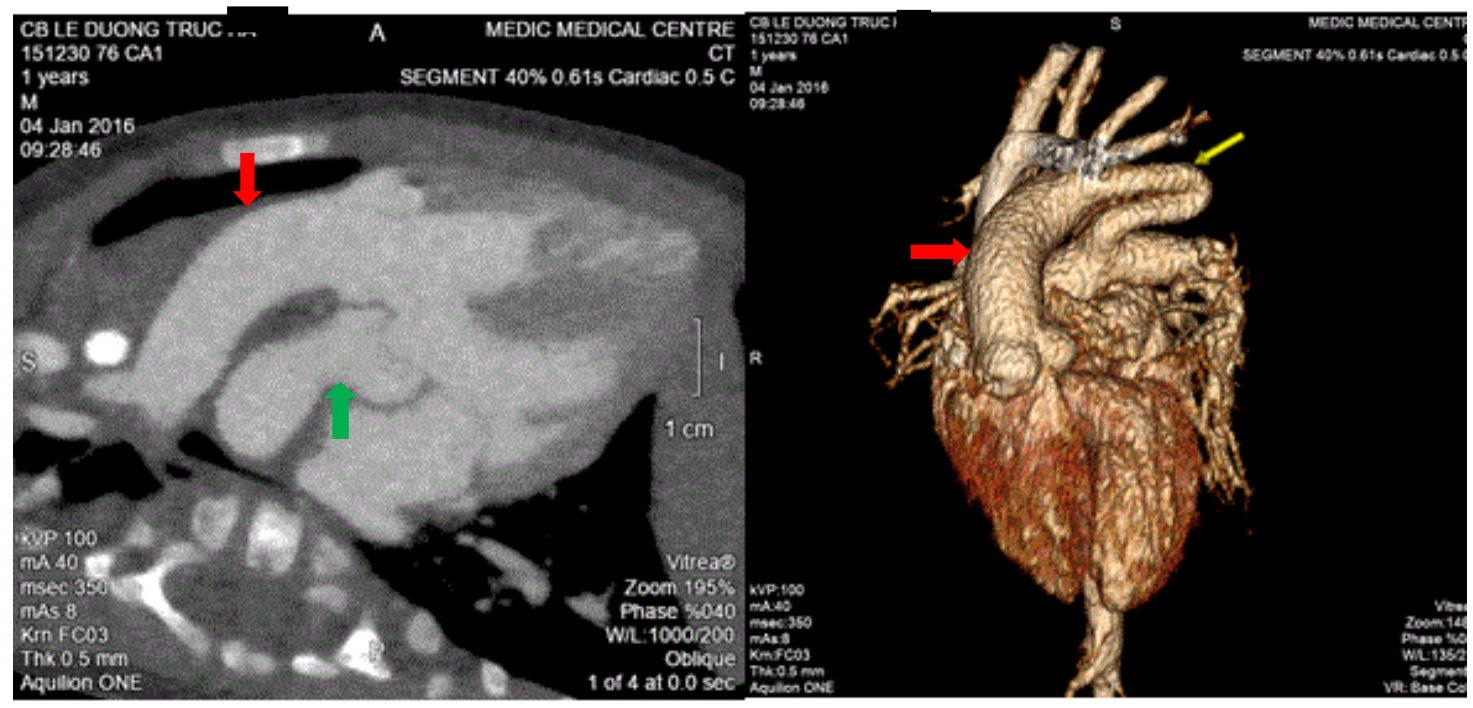

Figure 8. Transposition of great vessels; Aorta and pulmonary artery in parallel; 3D image : Aorta and Pulmonary artery switched, aorta in front of pulmonary artery

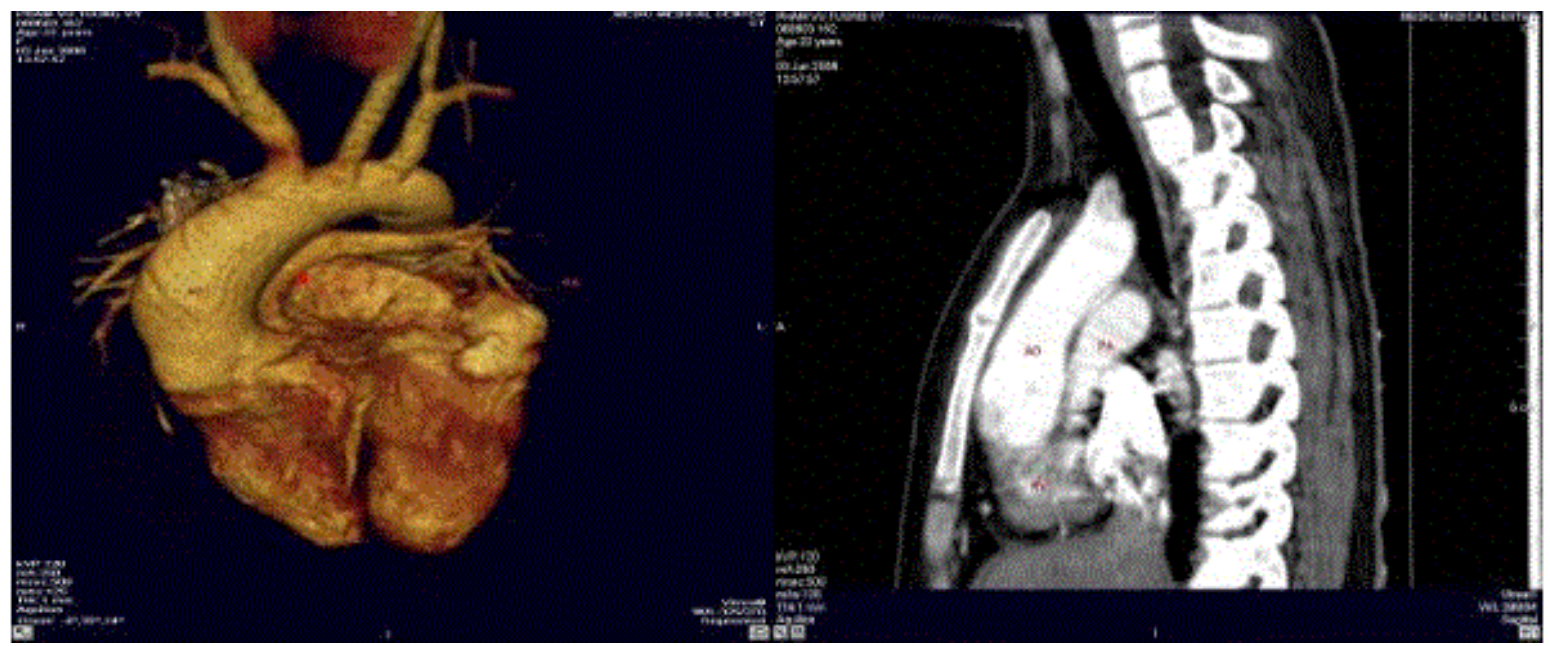

Figure 9. Double outlet right ventricle; Both of great arteries (aorta and pulmonary artery) arise from right ventricle and aorta is in front of pulmonary artery 


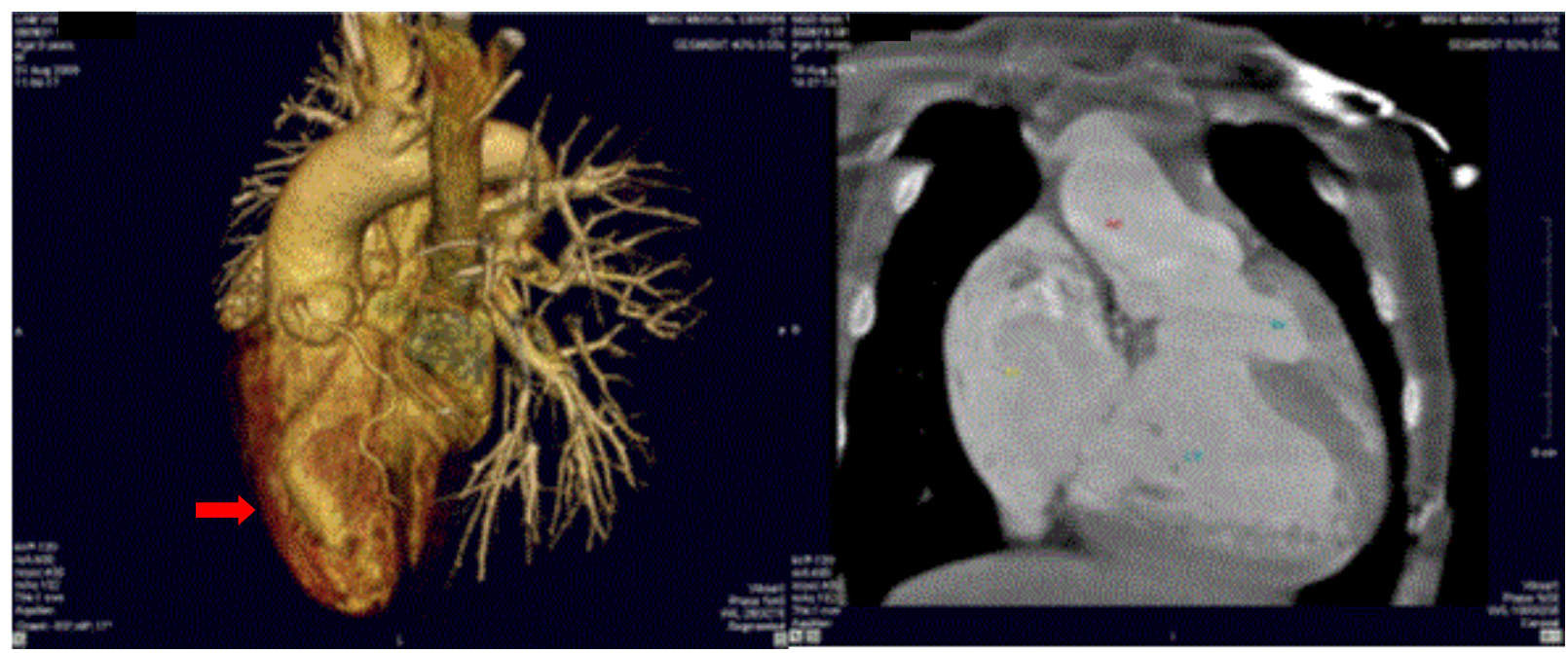

Figure 10. Single ventricle; Single ventricle- main right ventricle and left hypoplastic ventricle

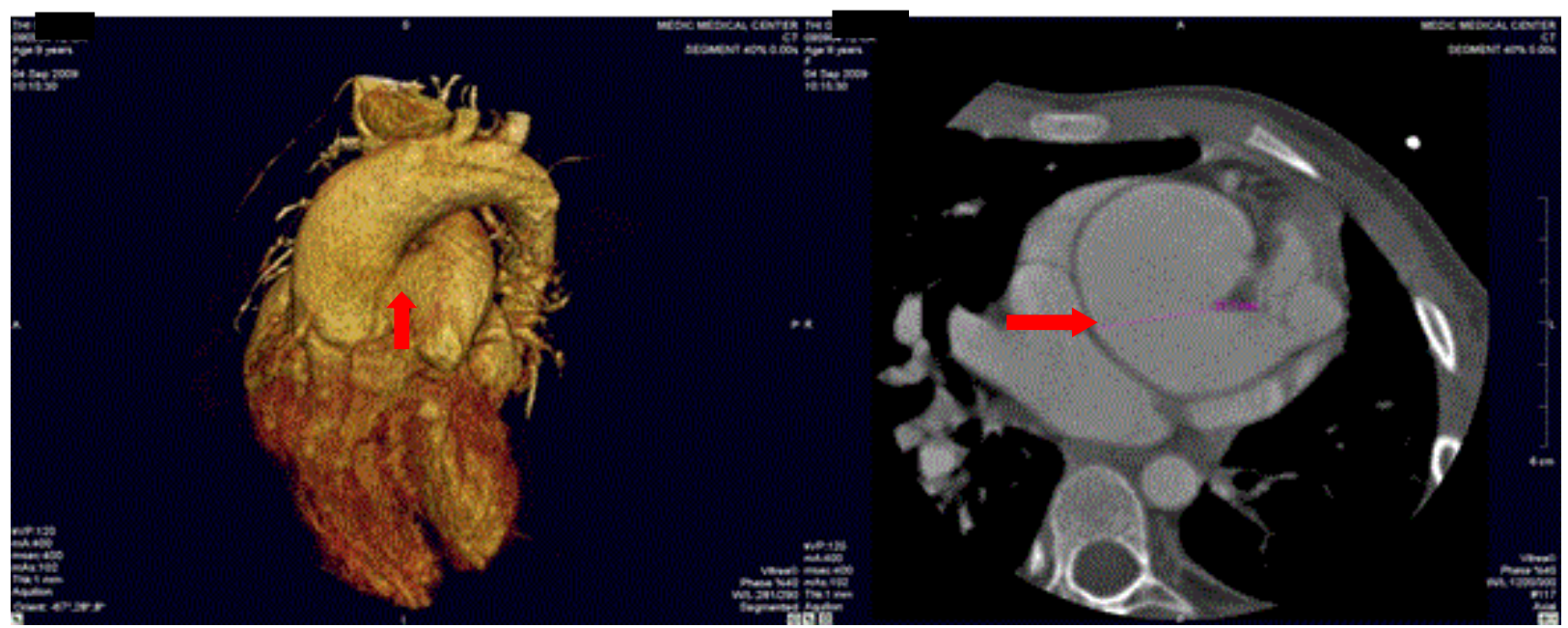

Figure 11. Aortopulmonary window; Axial view: A hole between aorta and pulmonary trunk

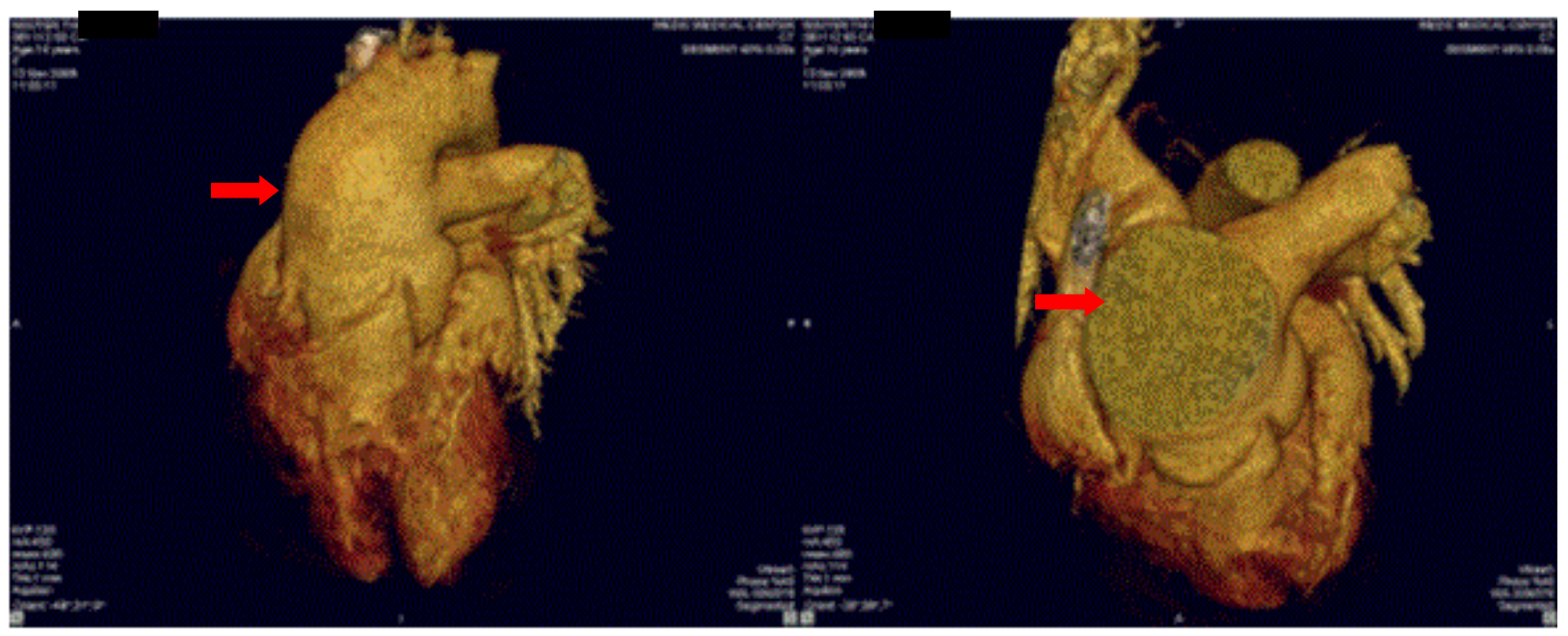

Figure 12. Truncus arteriosus; 3D images: Right and left pulmonary arteries arise from lateral aspects of truncal root reasonably distant from each or 


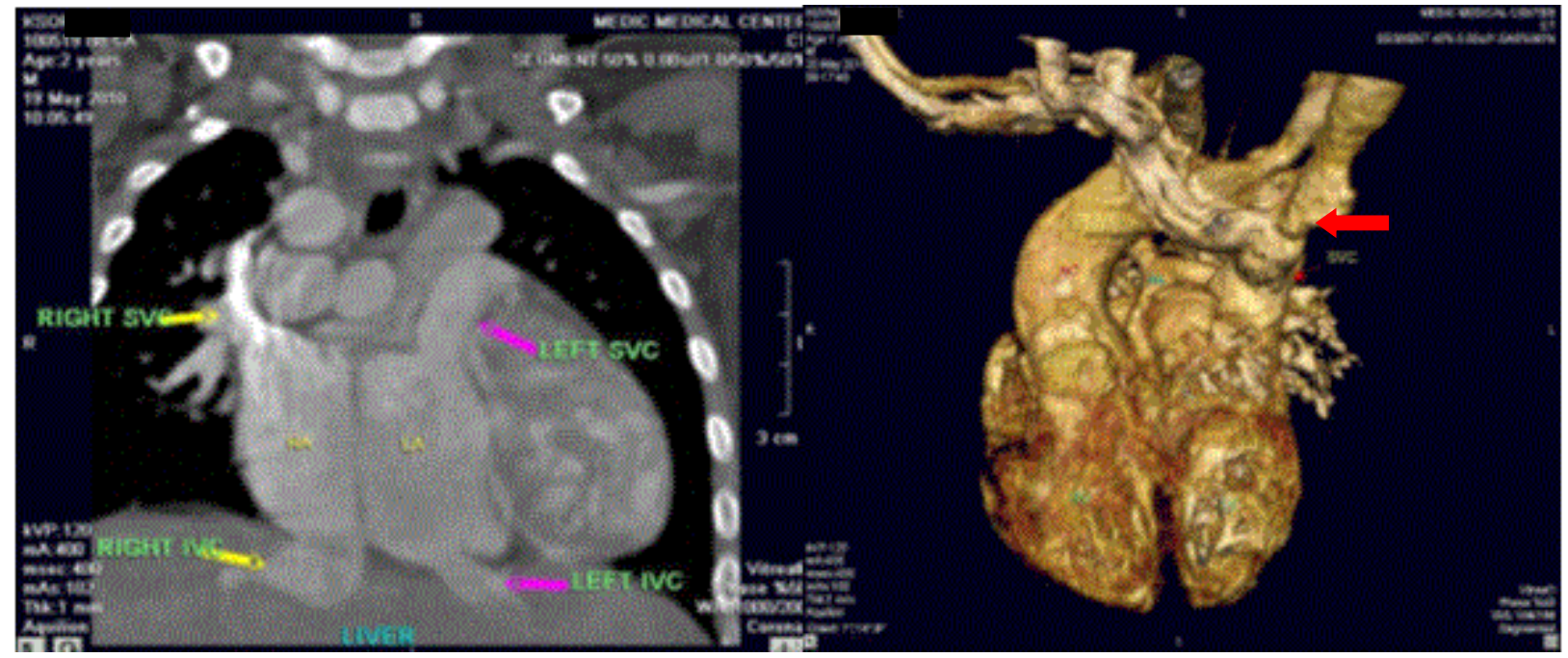

Figure 13a. Anomalous systemic venous return; Coronal view: Left superior vena cava connected to left atrium and hepatic vein connected to left atrium. Right superior vena cava or inferior vena cava draining into right atrium

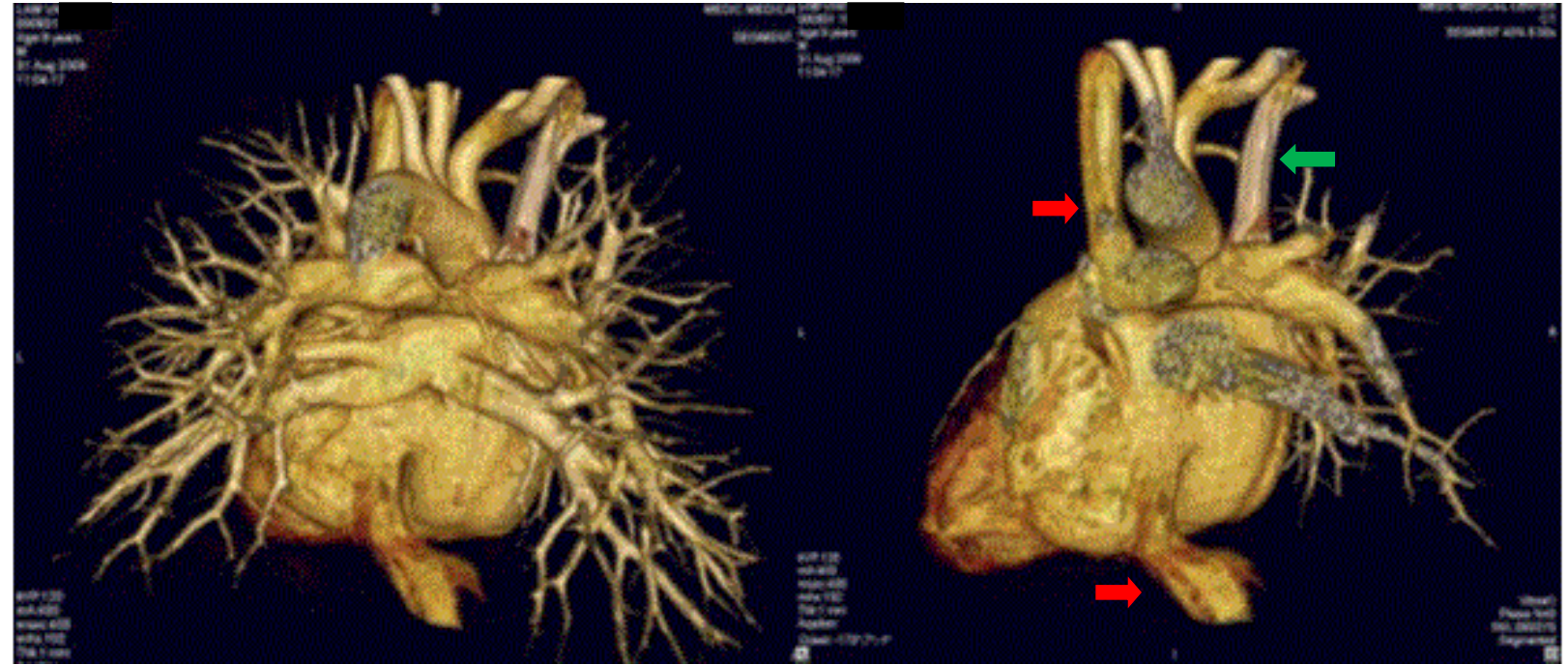

Figure 13b. 3D images : Left superior vena cava and inferior vena cava draining into left atrium

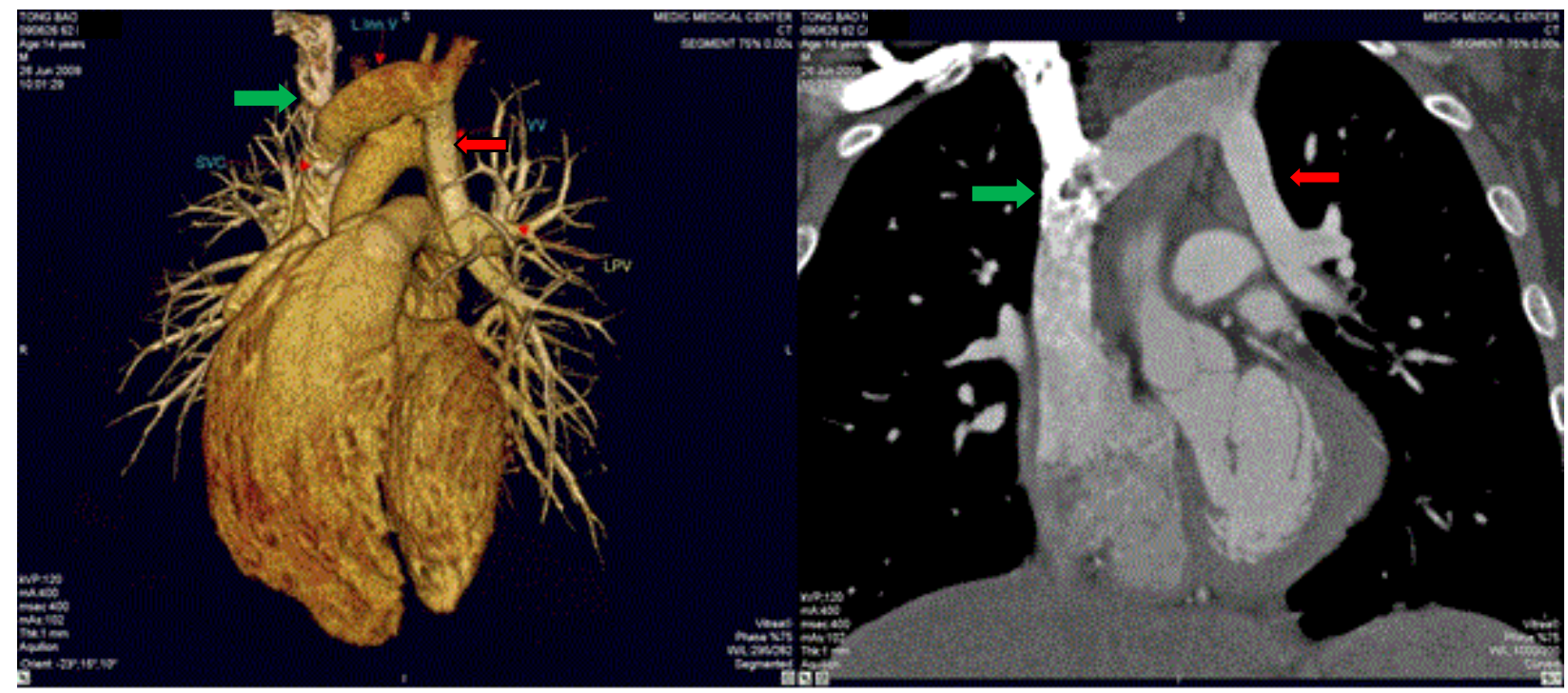

Figure 14a. Anomalous pulmonary venous connection; 3D image and coronal view: Drainage of left upper pulmonary vein (LUPV) via a vertical vein to innominate vein and right superior vena cava 


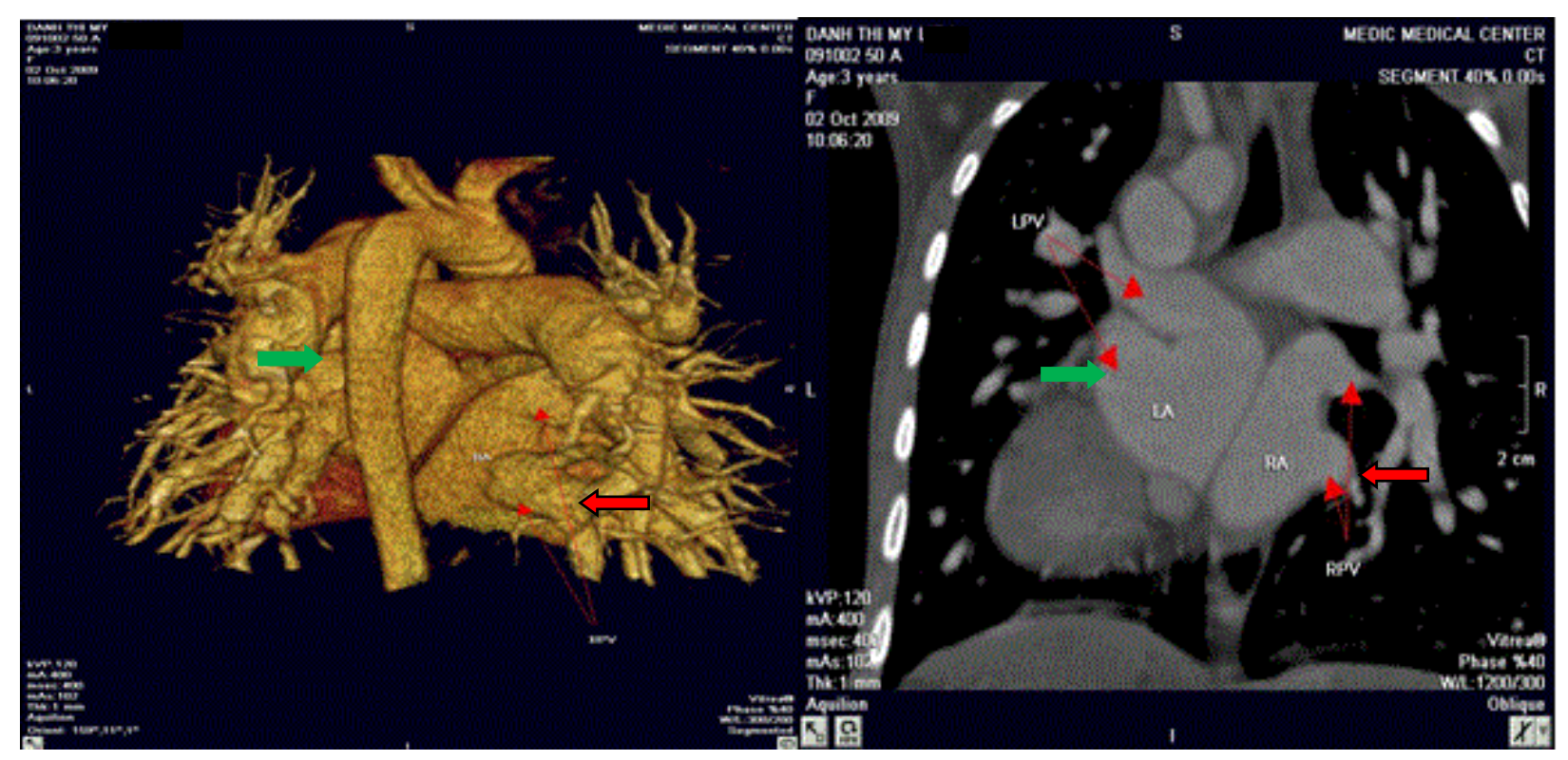

Figure14b. 3D images: Drainge of right upper and lower pulmonary vein to right atrium

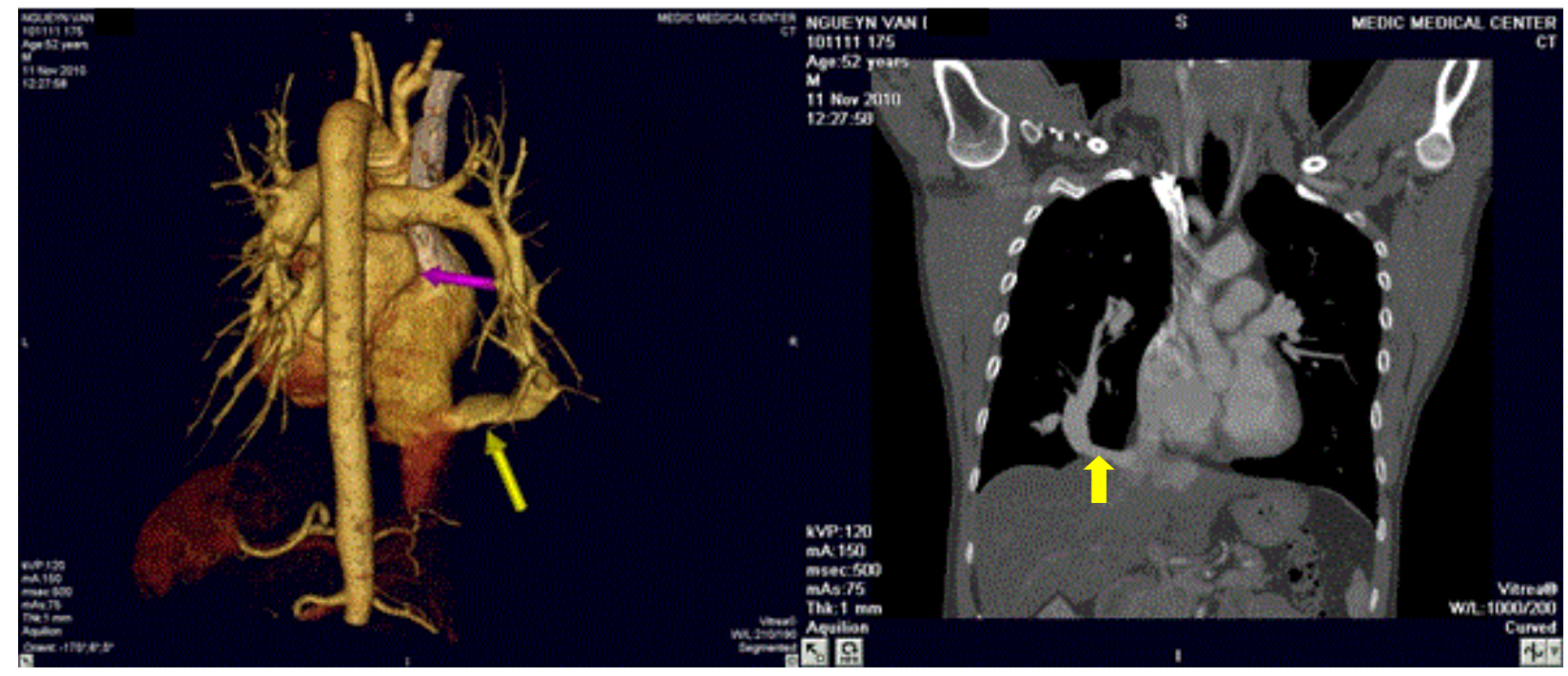

Figure 14c. 3D images: Drainage of right lower pulmonary vein (RLPV) to inferior vena cava (IVC)

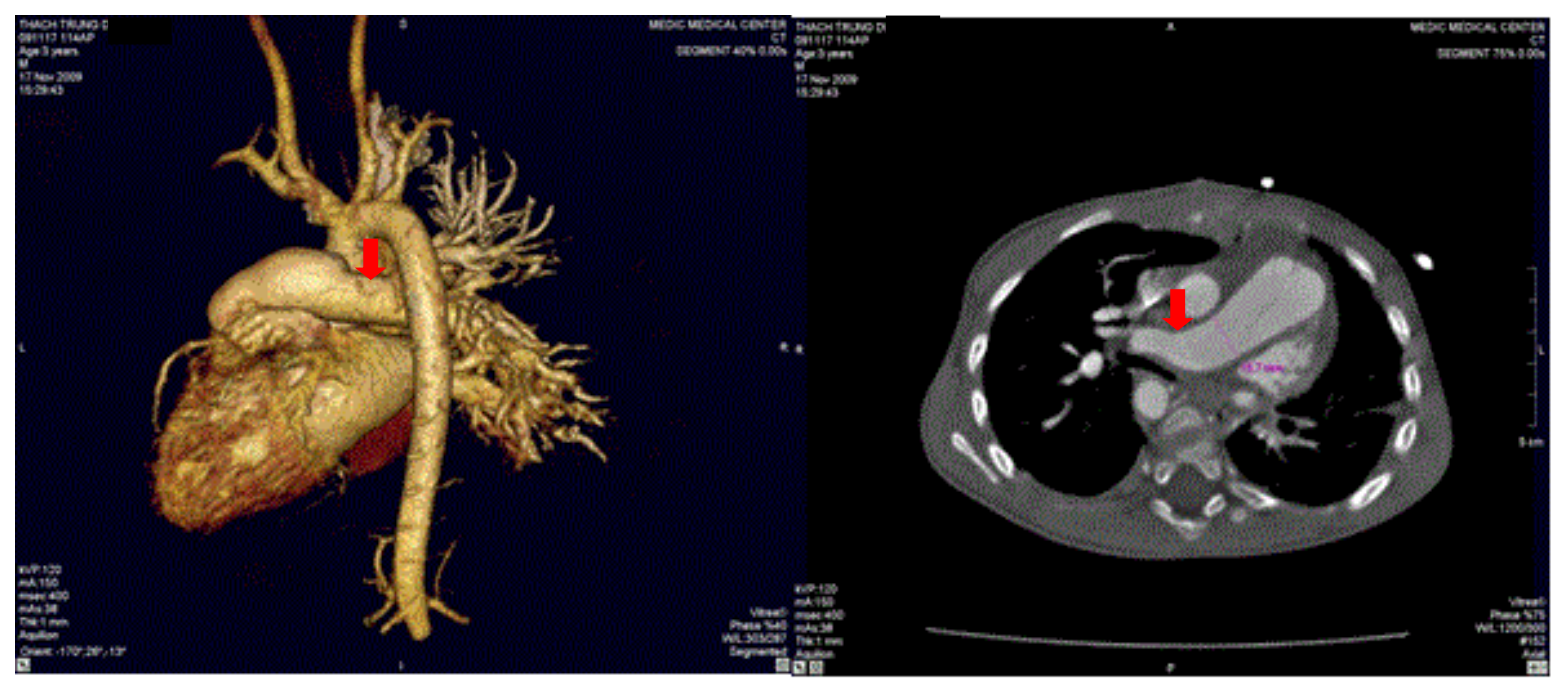

Figure 15. Single pulmonary artery; 3D image and Axial view: Seeing right pulmonary artery and absent left pulmonary artery 


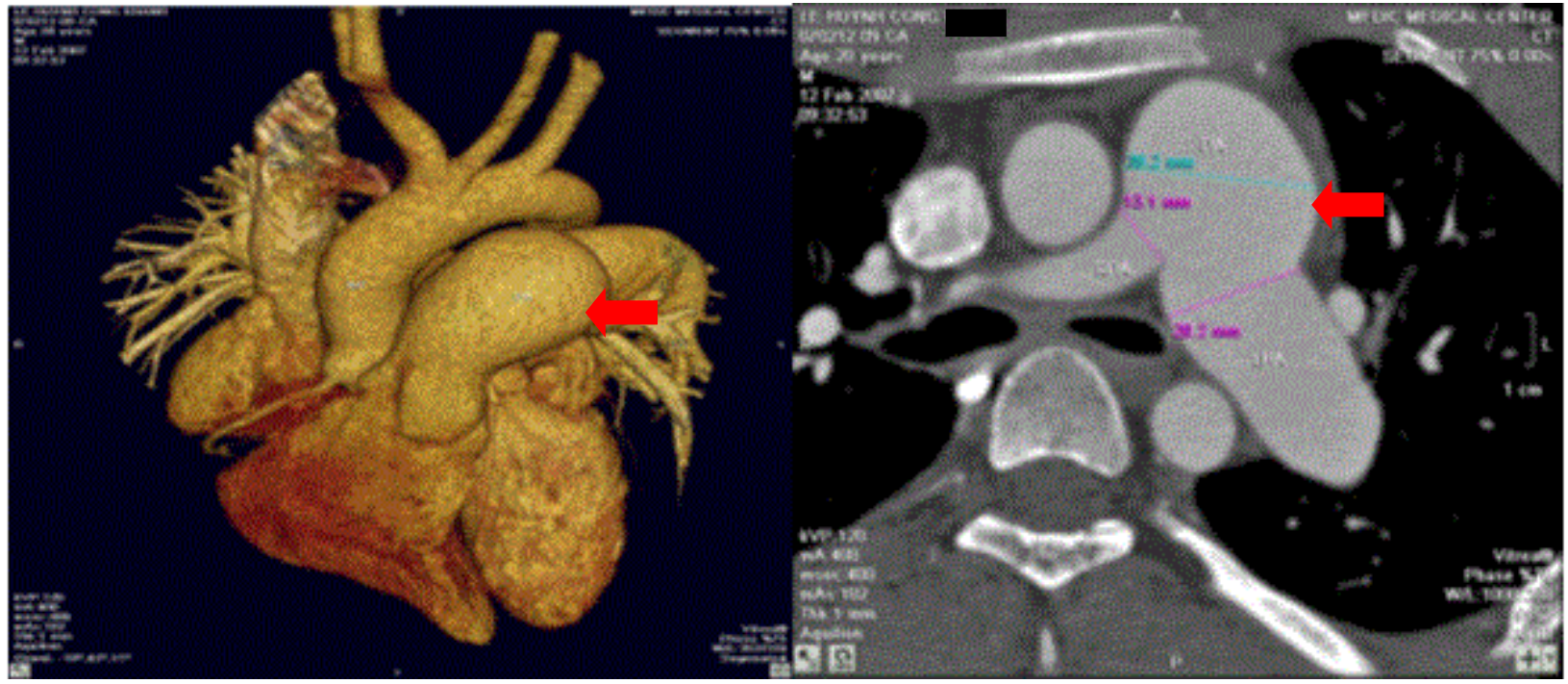

Figure 16. Pulmonary artery trunk aneurysm; D image and Axial view: Pulmonary artery trunk and left pulmonary artery aneurysm

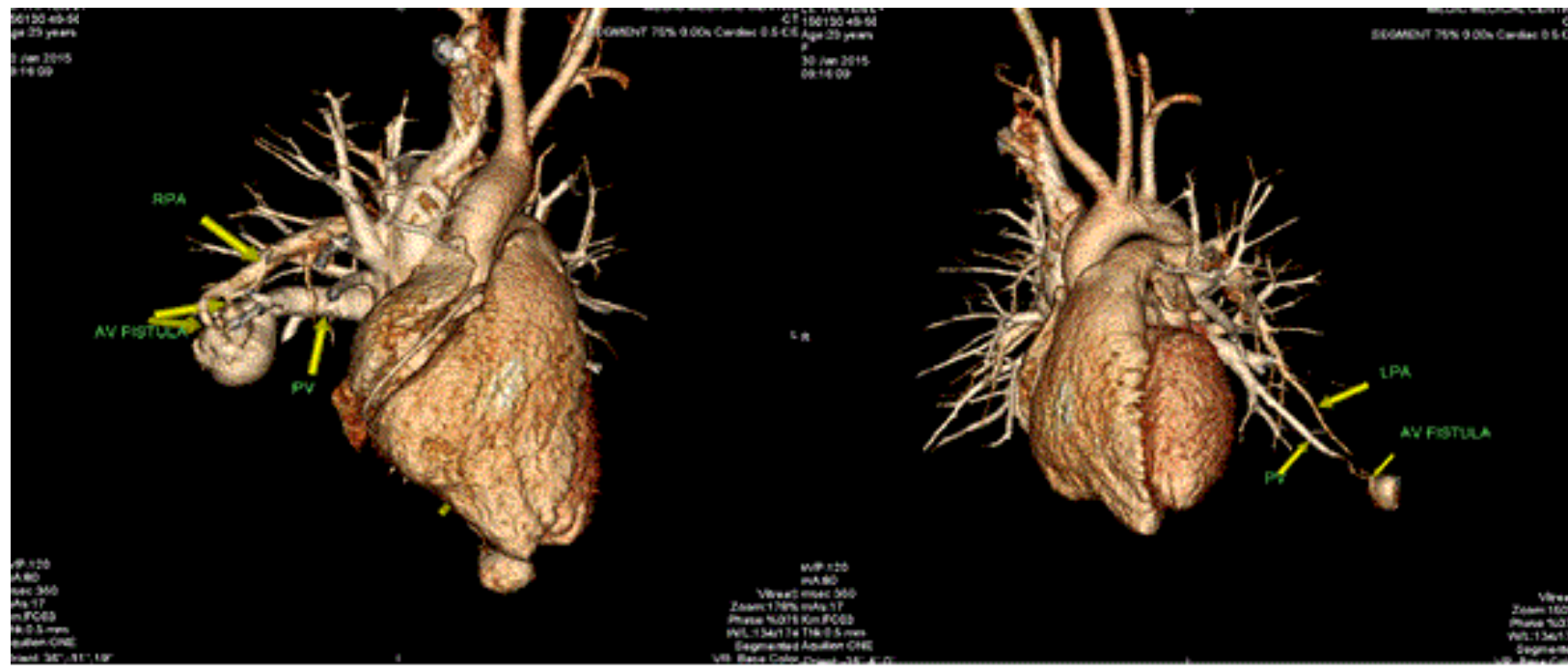

Figure 17. Congenital pulmonary arteriovenous malformation; 3D view: Right-to-left shunt between the pulmonary and vein

- To inject contrast by intravenous right hand.

- Contrast dose used $1 \mathrm{~mL} / \mathrm{kg}$.

- Drug pump speed depends on patient status and disease.

- Vitrea software: Reconstructed images by MPR, MIP and VRT.

- Effective radiation dose is low (320-MDCT is $3.69 \pm 061 \mathrm{mSv} ; 64$ MDCT is $12-14 \mathrm{mSv}$ ).

\section{Data analysis}

- The prospective study and case series report compare with operative and interventional angiography.

- Data collection at the HCM city Heart Institute, Tam Duc Heart Hospital and Medic medical centre.

\section{Results}

There are 658 operated cases, most of operated cases demonstrated the exact diagnosis of MDCT in congenital heart diseases (Figures 2-17).

\section{Discussion}

Congenital heart diseases associated with more malformations, complex aortopulmonary collaterals and anomalous coronary artery.

Echocardiography is the initial evaluative method for pre- and postoperation congenital heart diseases but this method can be limited in complex cases.

Multi-detector computed tomography overcomes the limit of Echocardiography by multiplanar reconstruction (MPR) and volume rendered techniques (VRT) reconstruction.

Volume rendered techniques (VRT) reconstruction clearly demonstrates the relationship between the heart and great vessels.

\section{Conclusion}

Multi-detector computed tomography is the fast and non-invasive diagnostic method with the high accuracy. Overcomes the limit of Echocardiography in complex congenital heart diseases. Provides the panorama and useful information's prior to the surgery. 


\section{References}

1. Michael H (2016) Crawford: Current diagnosis \& treatment in cardiology, chapter 27.

2. Gilkeson RC, Ciancibello L, Zahka K (2009) Multidetector CT Evaluation of Congenital Heart Disease in Pediatric and Adult Patients. AJR p.193.

3. Newswise Medical News: New CT Scanner for Early Detection of Heart Disease.
4. RSNA (2004) Effectiveness of Cardiac-Gated MDCT for the Diagnosis of Congenital Heart Disease in Small Children with High Heart Rates.

5. Budoff MJ, Shinbane JS, Achenbach S (2010) Cardiac CT Imaging. Diagnosis of Cardiovascular Disease.

6. Matthew j, Budoff, Stephan Achenbach and Jagat Narula: Atlas of cardiovascular computed tomography, chapter 11

Copyright: $\mathbb{C} 2019$ Son DP. This is an open-access article distributed under the terms of the Creative Commons Attribution License, which permits unrestricted use, distribution, and reproduction in any medium, provided the original author and source are credited. 